\title{
Instructions matter: Individual differences in navigation strategy and ability
}

\author{
Alexander P. Boone ${ }^{1} \cdot$ Bryan Maghen $^{1} \cdot$ Mary Hegarty $^{1}$ \\ Published online: 17 May 2019 \\ (C) The Psychonomic Society, Inc. 2019
}

\begin{abstract}
Individual differences in navigation strategy in the dual-solution paradigm (DSP) indicate that some people prefer to take learned routes, while others prefer to take shortcuts (Boone, Gong, \& Hegarty, Memory \& Cognition, 46, 909-922, 2018; Marchette, Bakker, \& Shelton, Journal of Neuroscience, 31, 15264-15268, 2011). Although work using the DSP has highlighted biases toward certain navigation strategies within individuals, a question remains as to why navigators do show a bias. Here, we questioned whether a bias toward navigation by learned routes indicates inability to take shortcuts, or whether other factors are at play, such as task demands and preferences. In two experiments, we began to untangle the association between the ability to construct survey knowledge, which is necessary if one is to take shortcuts (i.e., competence), and navigation strategy (i.e., performance) in virtual-reality navigation. Participants learned a route through a virtual environment and then navigated to goal locations in two experimental sessions. In Experiment 1, each participant navigated under two different instructions ("go to the goal" vs. "take the shortest path to the goal"), whereas in Experiment 2 two groups navigated under the same instructions in both sessions. Converging results from these experiments indicated that participants used more shortcuts overall under the shortcut instructions, and that this was not attributable to additional task exposure. Men were more likely to take shortcuts, under both the standard and shortcut instructions. This work indicates that the use of learned routes in the DSP does not necessarily imply that a person cannot take a shortcut, highlighting a dissociation between strategy and ability.
\end{abstract}

Keywords Navigation $\cdot$ Individual differences $\cdot$ Strategy $\cdot$ Efficiency $\cdot$ Sex differences

When navigating to a goal location, you have several choices of how to get there depending on factors such as how well you know the environment, your confidence in that knowledge, your specific goals, and time constraints. If you know the environment well and are in a hurry, you might choose to take a shortcut. Alternatively, you might take the way that you first learned or your habitual route. Whatever path is ultimately chosen, it is clear that knowledge of the environment was used to make decisions to execute your goal. However, taking that well-known path does not necessarily imply that you had no knowledge of alternative, potentially more efficient routes. This work explores individual differences in navigation strategy in the context of differing task instructions, in order to tease apart the influence of what someone can do (i.e.,

Alexander P. Boone

alexanderpaulboone@gmail.com

1 Department of Psychological and Brain Sciences, University of California, Santa Barbara, CA, USA navigation ability or competence) versus what someone actually does (i.e., strategy or performance) during a navigation task.

Before we can navigate effectively within an environment, we must build up knowledge about the overall environment. There are large individual differences in how people learn and subsequently express their knowledge of their environments. After learning a route through an environment, some individuals show good knowledge of the configuration of the environment, indicating that they have inferred survey (or configural) knowledge of the environment from the experience of following a route. For example, they are accurate at pointing to locations in the environment that are not visible from their current location (Ishikawa \& Montello, 2006; Schinazi, Nardi, Newcombe, Shipley, \& Epstein, 2013; Weisberg, Schinazi, Newcombe, Shipley, \& Epstein, 2014). However, the same research studies have indicated that some people, given the same experience of a route, do not develop survey knowledge, even after many experiences of the routes, suggesting that they have encoded the route but have not inferred survey knowledge. 
There are also large individual differences in how people navigate through a known environment, unveiling different navigation strategies. Marchette, Bakker, and Shelton (2011) developed the dual-solution paradigm (DSP) to study these strategies. In this task, participants learn a virtual maze on a desktop computer in the firstperson perspective. Participants are taken on the same prescribed route through the maze several times to learn the location of landmarks within the maze. After learning, participants are placed in locations within the maze and asked to navigate to previously learned goal locations. Each trial is constructed such that taking the learned route is effective but less efficient than taking a shortcut to the goal. Marchette et al. computed a measure of strategy equal to the number of trials on which the goal was reached by a shortcut divided by the number of trials on which the goal was reached (by either a shortcut or the learned route). This is referred to as the solution index and expresses a participants bias toward a given strategy. At the behavioral level, Marchette et al. found that participants varied widely in strategy, such that some participants took only the learned routes, others took shortcuts on all trials, and many others split their paths between learned routes and shortcuts, suggesting that they could use either strategy. Success in navigating to the goal in the DSP was not related to strategy (Marchette et al., 2011; Weisberg \& Newcombe, 2016), suggesting that successful navigation can be achieved by either strategy.

Prior research has indicated that these navigation strategies are supported by different brain areas. The hippocampus has long been thought of as the site of the cognitive map or survey knowledge of the configuration of an environment (Maguire et al., 1998; Maguire, Woollett, \& Spiers, 2006; O'Keefe \& Nadel, 1978), whereas the caudate nucleus supports the creation of stimulus-response associations for sequences of actions at relevant locations (Hartley, Maguire, Spiers, \& Burgess, 2003; Iaria, Petrides, Dagher, Pike, \& Bohbot, 2003) that are characteristic of familiar routes. Behavior in the DSP has been linked to these navigationally relevant brain areas, such that increased hippocampal activity during learning is associated with taking shortcuts at retrieval, and increased caudate nucleus activity during learning is associated with taking learned routes at retrieval (Marchette et al., 2011). Moreover, brain activation during encoding predicts the brain activation during subsequent navigation (Furman, ClementsStephens, Marchette, \& Shelton, 2014).

Although work using the DSP has highlighted biases toward certain navigation strategies within individuals, a question remains as to why navigators show a bias. One possibility is that the strategy bias reflects individual differences in the ability to construct a survey representation from route experience. Where taking a learned route requires memory for that route, taking a shortcut requires knowledge of the configuration of the environment - that is, survey knowledge, which must be inferred from the experience of taking a route. ${ }^{1}$ People who take learned routes may do so because they are less able to construct survey knowledge of the environment, and in the absence of a high-quality survey representation, they fall back on route knowledge. However, a second possibility is that a navigator might have survey knowledge necessary to execute a shortcut, but while performing the task might choose not to use that knowledge and to take the well-learned route instead. For example, she or he might feel that attempting to take the shortcut is risky, or might not have complete confidence in her or his ability to execute the shortcut. A third possibility is that a participant might think that the task is to take the learned route. These alternatives call to mind the distinction between competence and performance (cf. Chomsky \& Halle, 1965) and suggest that performance in the DSP, particularly as it relates to taking well-known routes, may not always be commensurate with the ability to construct survey knowledge, and someone's navigation strategy may not fully reflect their navigation ability.

If the ability to construct survey knowledge is the main determinant of using a shortcut strategy in the DSP, then more use of the shortcut strategy (a higher solution index) should be strongly correlated with the ability to construct survey knowledge. However, recent research has shown a dissociation between measures of survey knowledge and navigation strategy. Weisberg and Newcombe (2016) used the DSP in conjunction with a separate task in which participants learned two different routes through a virtual environment. After learning these routes, participants were placed at various locations on each route and asked to make pointing judgments to locations both within each route and between the routes. Accurate betweenroute pointing requires integrating the two routes and is considered to be a measure of survey (i.e., configural) knowledge. Weisberg and Newcombe showed that navigators with better pointing ability also found more goal locations in the DSP; however, pointing ability did not predict navigation strategy, as would be expected if the ability to construct survey knowledge was the main determining factor in using the shortcut strategy. Moreover, to date, only modest correlations have been found between the solution index and other measures of spatial ability, such as perspective taking, which is also associated with the ability to construct survey knowledge. Where Marchette et al. (2011) found that the correlation between strategy in the DSP and a perspective-taking

\footnotetext{
${ }^{1}$ By survey knowledge, we do not imply that the internal representation is metrically accurate, only that it contains information that allows one to compute routes through the maze other than the one you experienced. For example, such knowledge might come from a labeled graph, a type of representation proposed by Chrastil and Warren (2015) that encodes networks of paths between landmarks, with labels on the graph representing rough distances and directions.
} 
task was moderate $(r=.37)$, Boone, Gong, and Hegarty (2018) found nonsignificant correlations (.02 and .03 in two experiments) between these variables.

Taken together, these results suggest that a question remains as to whether people who take learned routes in the DSP do so because of a disinclination to take shortcuts, an inability to do so, or other factors, such as the experimental task demands. In previous administrations of the DSP, in an effort to understand navigation strategy biases, participants were asked to navigate to a specific goal but were never told how to arrive there. Here we investigated the difference between performance in the DSP (what someone does) and navigation competence (what someone can do). Specifically, we examined the effects of directly instructing participants to take a shortcut at the test phase, as compared to the standard instructions for the DSP. We predicted that a specific task instruction to take a shortcut would increase the use of shortcuts.

We also examined how sex differences in the DSP are affected by the task instructions. Previous work has indicated sex differences in self-report measures of navigation strategy (Lawton, 1994), such that men report more shortcut use and women report more use of learned routes and also more navigation anxiety. Sex differences are also found in objective measures of navigation efficiency, such as the time to escape from a maze (Moffat, Hampson, \& Hatzipantelis, 1998; Sandstrom, Kaufman, \& Huettel, 1998). In a recent study using the standard DSP, Boone et al. (2018) found that men took significantly more shortcuts than women, whereas women sometimes took more learned routes than males. Another goal of the present research was to determine the extent to which these sex differences in strategy were due to differences in ability to take a shortcut (competence) or to differences in strategy preference (performance). Therefore, we also investigated whether sex differences are present in this task when people are given the standard instructions versus when they are instructed to take shortcuts.

\section{Experiment 1}

In Experiment 1, participants learned and were tested in two virtual maze environments. In the first session, after learning a route through a virtual maze, participants were placed at different locations in the maze and asked to navigate to specific goals on each trial (e.g., "Please navigate to the harp."), as in the standard version of the DSP. During the second session, participants learned a second maze and were subsequently asked to take the shortest path to different goal locations (e.g., "Please take the shortest path to the swing."). After this session, participants also completed a landmark placement task, in which they had to identify the landmarks at different locations in the maze. This task is considered to be a measure of the quality of participants' environmental knowledge.
If the solution index (the measure of navigation preference/ bias in the DSP) reflects the ability to construct survey knowledge alone, then there should be little or no change in shortcut behavior due to instructions and navigation strategy. If strategy is not completely determined by this ability, then we might see a shift in initial biases toward more shortcuts when participants are specifically asked to take shortcuts. We also examined how completeness of knowledge of the maze (as measured by a landmark placement task) was related to solution strategy. Furthermore, as in previous research, we expected men to take more shortcuts with the standard "go-to-goal" instructions; however, if these standard instructions reflect aspects of strategy choice other than ability (e.g., risk taking and experimental demand), then women might show performance more similar to that of men when they are told to take shortcuts.

\section{Method}

\section{Participants}

The participants were 36 University of California, Santa Barbara, undergraduates participating in return for course credit. Four of the participants (three female, one male) were excluded from the analysis due to motion sickness. Thirty-two participants (16 females, 16 males) were included in the final analyses. With this sample size, the power to detect a medium effect of instructions $\left(\eta_{\mathrm{p}}{ }^{2}=.06\right)$ at $p=.05$ was .81 .

\section{Design}

This experiment had a 2 (session: first vs. second) $\times 2$ (Maze 1 vs. Maze 2$) \times 2($ Sex: male vs. female) mixed experimental design. All participants were given the standard DSP instructions ("go to goal") in the first session and were told to take a shortcut to the goal in the second session. Maze type and instructions were manipulated within subjects, while sex varied between subjects. Maze order was counterbalanced across participants.

\section{Materials}

The experiment was administered using a Dell XPS with a GeForce GTX 1070 graphics card. The task was presented using Unity3D and displayed on a 24-in. LCD monitor $(289.9 \times 531.4 \mathrm{~mm}$ display area $)$, with a refresh rate of $60 \mathrm{~Hz}$ at a resolution of $1,920 \times 1,080$. The viewing distance was approximately $1,000 \mathrm{~mm}$.

Maze development Maze and trial development were undertaken in a systematic manner in order to ensure equivalency between mazes in a number of dimensions, including the number of steps on the learning path, the number and type 
of objects within the mazes, and the distance to be traveled across trials on the learned route or the shortcut. Each maze environment was initially constructed in Blender (a free, opensource software toolset to create 3-D objects) and then imported into Unity3D. These mazes were constructed as 11 $\times 11$ grids of unit squares and extended one unit square upward. In this way, the number of squares, or "steps," traversed by a participant on a given trial can be counted.

As can be seen in Fig. 1a, Maze 1 was taken directly from Marchette et al. (2011) without modification of the environmental structure, except for differences in textures and objects. Maze 2 (Fig. 1b) was created by mirroring Maze 1 and then placing the starting point of the route in a different location. Additionally, the texture of the walls was different in each maze, to further suggest a change of environment. In Maze 1, the walls consisted of red brick (Fig. 1c), whereas gray bricks were used in Maze 2 (Fig. 1d). The floor texture (basic light gray speckled asphalt) and sky (basic light blue, no single salient light source) were unchanged between the environments. A training maze was also created, to allow participants time to understand the navigation controls before starting the task. This

a)

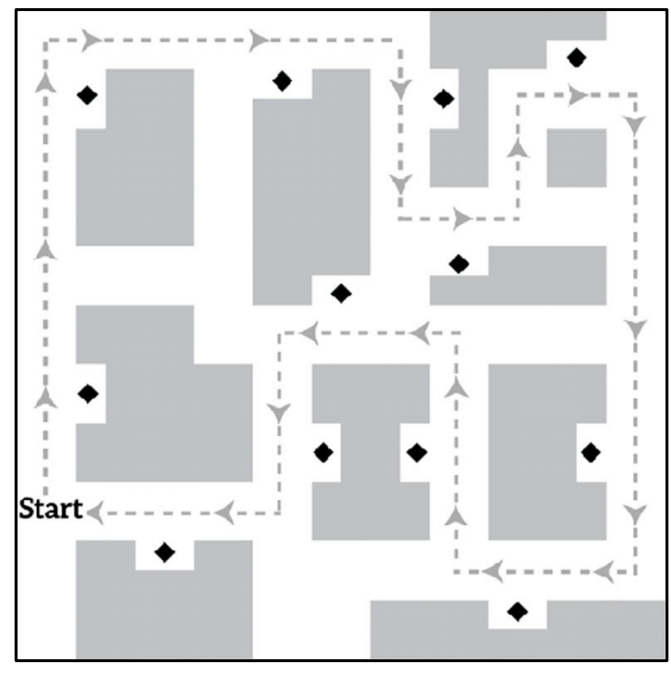

c)

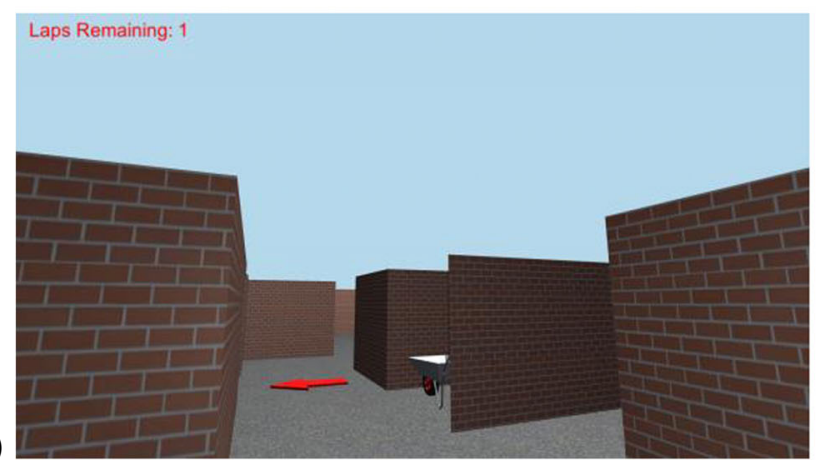

Fig. 1 (a) Maze 1 schematic structure, modeled after Marchette et al. (2011); (b) Maze 2 schematic structure, which mirrors Maze 1 and has a different start point to the route; (c) participant's view of Maze 1; and (d) Maze 2 during the learning phase, taken from the same viewpoint. In the maze was the same size and shape as the other mazes, and the walls were textured using the red brick. Inside the training maze were four square pylons arranged in a $2 \times 2$ grid, and participants practiced walking around the pylons.

Each maze contained 12 alcoves where landmarks were placed. In Maze 1, the order of the landmarks encountered along the original learning tour was as follows: a brown desk chair, a blue US Postal Service mail drop box, a multicolored telescope, a large potted plant, a picnic table, a stove, a piano, a trashcan, an empty bookshelf, a wheelbarrow, a harp, and a wooden wishing well. In Maze 2, the order of the landmarks was a desk, a water cooler, a streetlamp, a red stepladder, a refrigerator, a bicycle, a lion statue, a couch, a phone booth, a wooden swing, a grandfather clock, and a television. All objects were downloaded from Turbosquid.com or through the Unity asset store. The objects were visible throughout both learning and retrieval testing.

Trial selection Trials were chosen such that the shortcut on each trial was at least $25 \%$ shorter than the learned route. Participants also sometimes reversed the learned route, and

b)

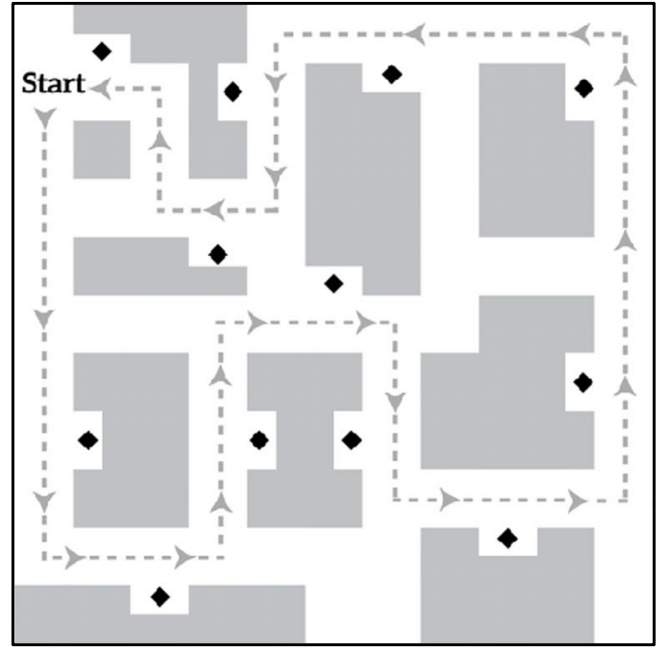

d)

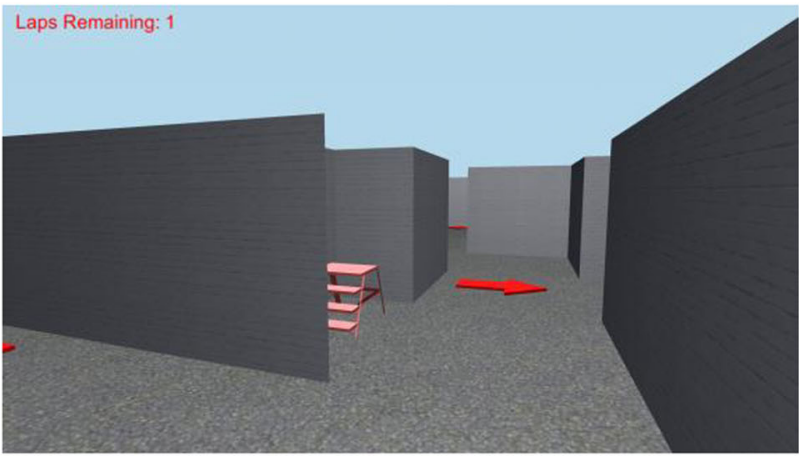

maps of the mazes, black diamonds represent the locations of landmarks (objects) that served as the goal locations in the navigation trials. Participants never saw a top-down view during learning 
the shortcut was also at least $19 \%$ shorter than reversing the learned route (Boone et al., 2018). There were 20 trials, but due to experimenter error, data were collected for only the first 12 trials in each maze. On average, the shortcuts were $51 \%$ of the length of the learned route, meaning that taking the shortcut across all trials would save just over half of the distance from taking the learned route.

Landmark placement task As an independent measure of environmental knowledge, a landmark placement task was included after the completion of all navigation task trials. After the navigation trials in the second session, a map was given to participants, indicating the location of each object within the maze, designated by a black diamond with a line, as can be seen in Fig. 2. The task was to write the name of the object in each location. The first object along the route was provided, and participants were not allowed to move the map in order to complete the task. This task was scored such that a correctly identified object location was one point, whereas an incorrectly placed item or an omission received zero points (maximum score $=11$ ). This task was administered only after the second set of trials, to avoid biasing or otherwise influencing performance on the second set of navigation trials.

Questionnaires Finally, participants completed several questionnaires that are beyond the scope of this article. They also completed a demographics questionnaire that included a question in which participants were asked to report how many hours they spend playing video games per week (never, less than $1 \mathrm{~h}$ per week, 2-3 h per week, 4-5 h per week, more than 5 h per week).

\section{Procedure}

After giving informed consent, participants practiced using the active navigation controls (keyboard and mouse) in the training maze. Next, participants were placed within one of the two experimental mazes (red or gray brick). Participants

\section{Participant Number:}

Instructions: The map below shows the layout of the environment you just learned. The diamonds indicate object locations. Please write the name of the object on the line for each location. The first one has been completed for you. You will have five minutes to complete this task.

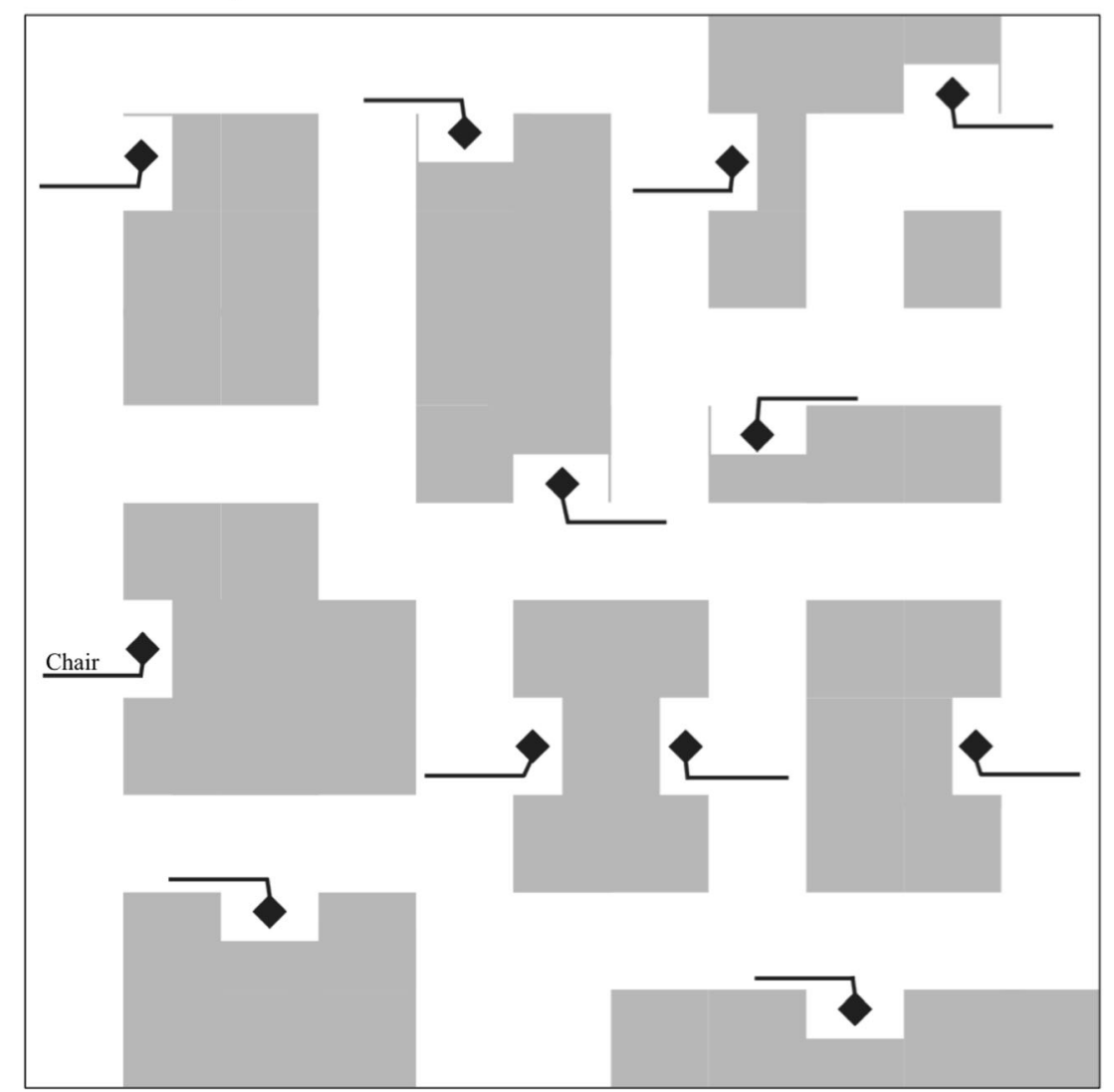

Fig. 2 Landmark placement task and instructions used in both Experiments 1 and 2, presented on $8.5 \times 11$ sheets of paper. Black diamonds represent the object locations. The first object encountered in each maze was marked. The maze presented is the original maze schematic. A mirrored schematic was used for the mirrored maze 
were asked to follow a route indicated by red arrows on the ground, saying the names of each object aloud as it came into view. Invisible barriers blocked each corridor that was not on the learning path, but the view of the corridor was not obscured. Participants traversed this route a total of five times. Following a short break, participants were given instructions for the testing phase, in which they were placed in front of an object on the learned route and asked to navigate to another object on the learned route. During the first set of trials (Session 1), participants were instructed to navigate to various goal locations around the maze and were told that they would have $40 \mathrm{~s}$ total to find the goal. Each trial concluded when the participant walked into the goal location or when $40 \mathrm{~s}$ had expired. After all trials had been completed in Session 1, participants were given a 5-min break. After the break, participants returned to the computer and learned the second maze. For the trials in the second session, participants were instructed, on each trial, to take the shortest path to the goal.

After the second set of navigation trials were completed, participants were presented with the landmark placement task for the second maze. Participants were given a total of $5 \mathrm{~min}$ to complete the task. Next, participants were administered an online questionnaire, including a self-rating of the frequency of their video game use and general demographic information. After the completion of all tasks, participants were debriefed and dismissed.

DSP strategy trial coding system We used the coding system established in earlier research (Boone et al., 2018), adapted from Marchette et al. (2011). To measure strategy, each trial for each participant was coded as being on the learned route, a shortcut, a reversal of the learned route, or wandering (longer than the learned route and repeating part of their own route). Furthermore, each trial was coded as a success or failure to reach the target, as a measure of navigation performance. As in previous research (Boone et al., 2018), some trials did not strictly fall in a single category, and cutoffs were used to categorize these trials (liberal coding). For trials coded as shortcuts, the participant's path was no more than $84 \%$ the length of the learned route and less than $70 \%$ of the path taken was on the learned route. The average length of trials liberally coded as shortcuts was $61 \%$ of the learned route, and the average overlap of the liberal shortcuts with the learned route was $38 \%$. Conversely, trials were coded as following the learned route if participants traversed $70 \%$ or more of the learned route. Trials liberally classified as learned route overlapped this route by $87 \%$, on average. Trials liberally coded as reversals of the learned route overlapped the reversed route by at least $70 \%$, with $80 \%$ reversal on average. Trials were classified as "wandering" when the participant crossed over their own route and the route taken was longer than the learned route. Finally, trials that did not meet any of these criteria were classified as uncodable. Using these data, we computed a measure equal to the number of shortcut trials (including liberally coded trials) over all successfully completed trials, producing a number on a scale of 0 (indicating no shortcuts) to 1 (indicating all shortcuts). ${ }^{2}$

Finally, navigation efficiency was assessed by computing an average path efficiency metric. A participant's path efficiency for each trial was the distance traveled on that trial divided by the length of the shortest possible path (shortcut) between the start and end locations. An individual's overall path efficiency was the average path efficiency across trials.

\section{Results}

\section{Navigation performance}

Descriptive statistics can be found in Table 1. Participants were highly successful in navigating to the goal in general; however, a 2 (session/instructions) $\times 2$ (gender) repeated measures analysis of variance (ANOVA) indicated that men $(M=$ $11.22, S D=1.02)$ were significantly more successful in reaching the target than were women $(M=10.16, S D=$ 1.68), $F(1,30)=6.80, p=.01, \eta_{\mathrm{p}}{ }^{2}=.19$. Neither the main effect of session (instructions) nor the interaction of session and gender on navigation performance was significant, all $F_{\mathrm{s}}(1,30) \leq .81, p \mathrm{~s} \geq .38$.

\section{Navigation strategy}

It was predicted that more shortcuts would be taken when participants were asked to take shortcuts than with the standard instructions in the second session. A 2 (session) $\times 2$ (gender) repeated measures ANOVA was conducted on the proportions of successful shortcuts (solution index). As can be seen in Fig. 3, we found a main effect of session, such that participants took more shortcuts in the second session (shortcut condition) $(M=.61, S D=.22)$ than in the control condition $(M=.36, S D=.24), F(1,30)=20.67, p<.001, \eta_{\mathrm{p}}{ }^{2}=.41$. Consistent with the results of Boone et al. (2018), a main effect of gender was also found, such than men took more shortcuts $(M=.58, S D=.23)$ than women $(M=.39, S D=$ $.20), F(1,30)=13.21, p<.001 \eta_{\mathrm{p}}{ }^{2}=.31$. However, there was no interaction between session and gender, $F(1,30)=0.37, p$ $=.55$.

\footnotetext{
${ }^{2}$ Previous work using the DSP had classified trials as either shortcut or learned routes and had used a solution index measure of shortcuts over shortcuts and learned routes. Boone et al. (2018) revealed other types of strategies that are used, besides learned routes and shortcuts, such as reversals of the learned route and wandering. Therefore, using shortcuts plus learned routes as the denominator meant that many successful trials were not counted. Our measure of shortcuts over all successful trials is equivalent to the Marchette et al. (2011) solution index, in that in their study almost all successful trials were classified as shortcuts or learned routes (with a small number of unclassifiable trials).
} 
Table 1 Descriptive statistics (means with standard deviations in parentheses) for solution index, proportions of trials that were successful, and proportions of trials on which each strategy was used in Experiments 1 and 2

\begin{tabular}{|c|c|c|c|c|c|c|}
\hline & \multicolumn{2}{|c|}{ Experiment 1} & \multicolumn{4}{|c|}{ Experiment 2} \\
\hline & \multirow{2}{*}{$\frac{\text { Control }}{\text { Session } 1}$} & \multirow{2}{*}{$\frac{\text { Shortcut }}{\text { Session } 2}$} & \multicolumn{2}{|l|}{ Control } & \multicolumn{2}{|l|}{ Shortcut } \\
\hline & & & Session 1 & Session 2 & Session 1 & Session 2 \\
\hline Solution index & $.36(.24)$ & $.61(.22)$ & $.30(.17)$ & $.35(.22)$ & $.46(.18)$ & $.51(.18)$ \\
\hline Successful trials & $.88(.13)$ & $.90(.11)$ & $.87(.13)$ & $.88(.11)$ & $.88(.10)$ & $.90(.09)$ \\
\hline Shortcut strategy & $.32(.23)$ & $.56(.24)$ & $.26(.16)$ & $.30(.20)$ & $.41(.18)$ & $.47(.18)$ \\
\hline Learned route & $.33(.29)$ & $.13(.16)$ & $.36(.22)$ & $.31(.24)$ & $.17(.15)$ & $.15(.13)$ \\
\hline Route reversal & $.10(.09)$ & $.09(.09)$ & $.12(.09)$ & $.12(.09)$ & $.10(.08)$ & $.08(.07)$ \\
\hline Wandering strategy & $.02(.04)$ & $.02(.04)$ & $.03(.04)$ & $.02(.03)$ & $.03(.04)$ & $.05(.05)$ \\
\hline Uncodable trials & $.11(.09)$ & $.09(.08)$ & $.09(.10)$ & $.13(.08)$ & $.17(.09)$ & $.16(.10)$ \\
\hline
\end{tabular}

Proportions of trials that were successful (i.e., the goal was reached) are reported, because there were different numbers of trials in each experiment. Solution index refers to number of shortcuts taken divided by number of successful trials. Shortcut, learned route, route reversal, wandering, and uncodable refer to the strategy coding expressed as a percentage of successful trials in each experiment

Figure 4 presents individual participants' solution index scores for each session of the task, as a function of change in the solution index. Each line represents the amount of change for a single participant across sessions, where the first session (control instructions) is denoted as a solid gray circle and the second session (shortcut instructions) is denoted as a dark gray triangle. Participants are ordered by their solution index in the control condition, and then by the amount of

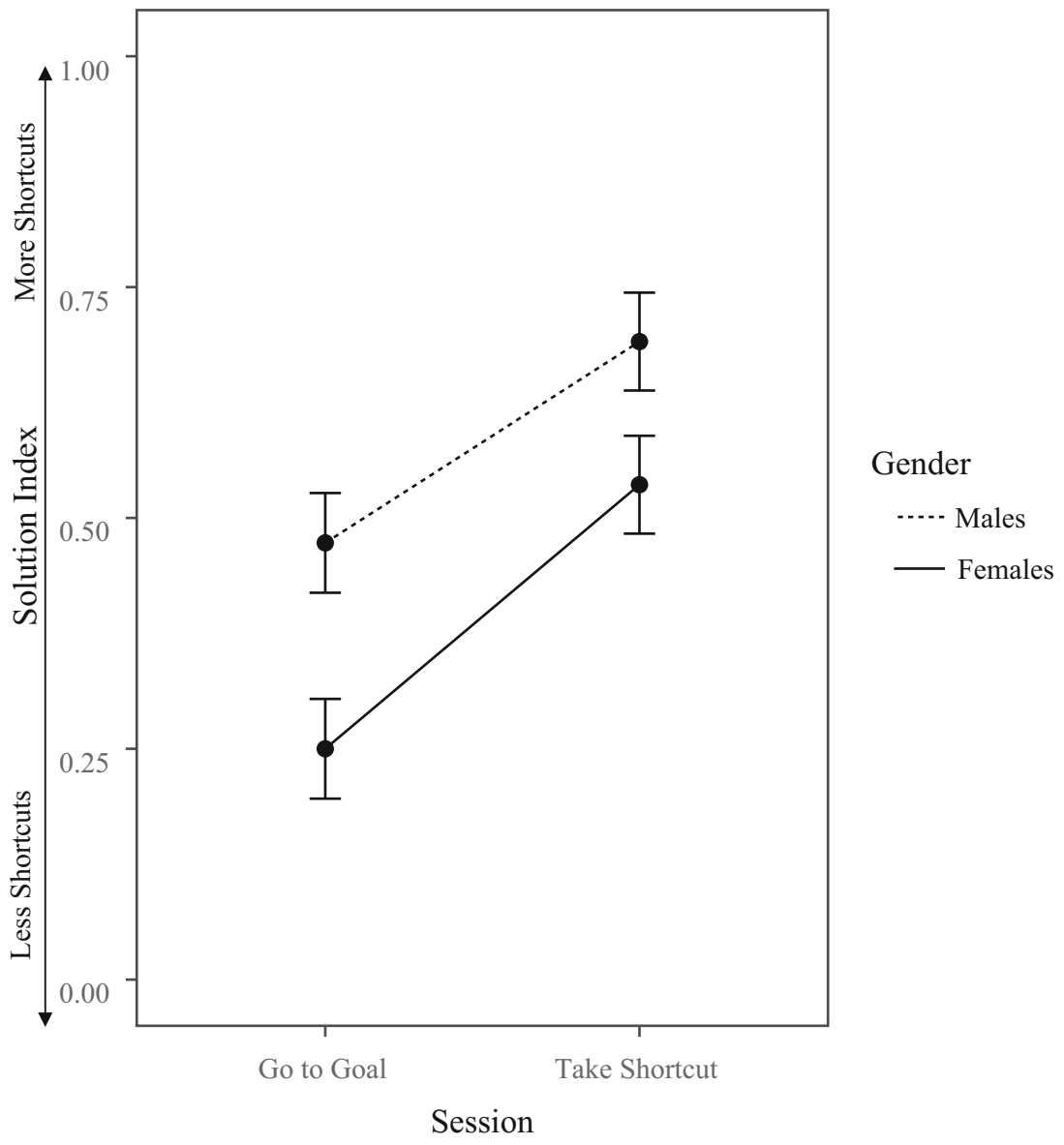

Fig. 3 Solution index by session and gender in Experiment 1. Error bars indicate standard errors. Larger values of the solution index indicate more shortcuts 


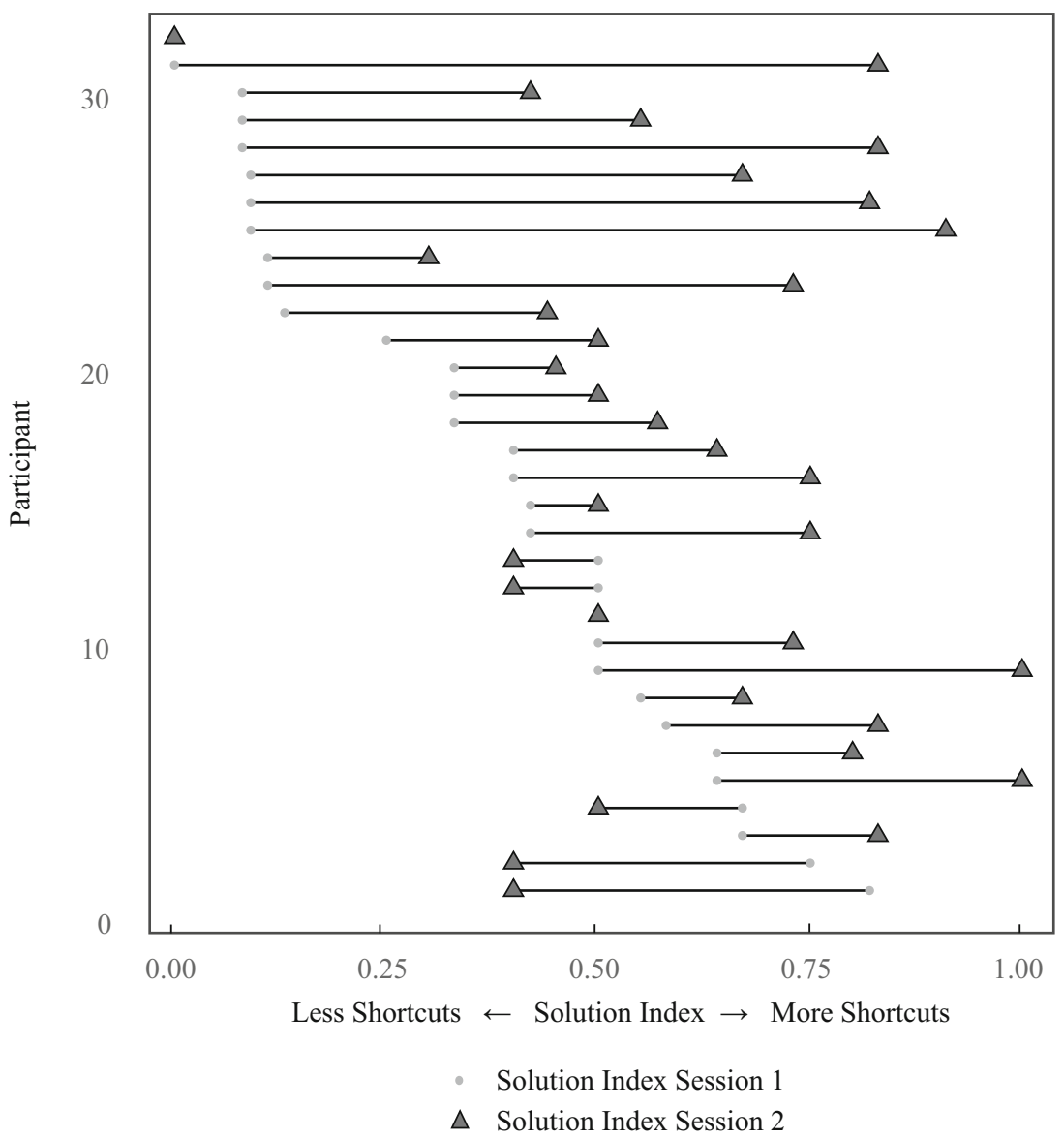

Fig. 4 Dumbbell plot showing changes in solution index as a function of each session of Experiment 1. The gray points represent each participant's solution index in the first session (control condition), and the gray triangles represent the solution index in the second session (shortcut condition)

change. As can be seen, most participants shifted toward taking more shortcuts (toward the right) in the second session $(n$ $=25)$, but a minority shifted toward fewer shortcuts $(n=5)$ and or did not shift $(n=2)$. The larger shifts were toward taking substantially more shortcuts.

\section{Navigation efficiency}

A 2 (session) by 2 (sex) repeated measures ANOVA on path efficiency ${ }^{3}$ indicated that participants were more efficient in the second session $(M=1.98, S D=.38)$ than in the first $(M=$ $2.42, S D=.54), F(1,30)=22.17, p<.001, \eta_{\mathrm{p}}{ }^{2}=.43$, but notably, even in the second session, participants were taking paths that were on average about twice the length of the shortcut. Men $(M=1.98, S D=.39)$ were more efficient that women $(M=2.41, S D=.42), F(1,30)=15.74, p<.001, \eta_{\mathrm{p}}{ }^{2}=.34$, and the interaction was not significant, $F(1,30)=1.89, p=.18$.

\footnotetext{
${ }^{3}$ In Experiment 1, no differences were found when using time efficiency over path efficiency. However, in Experiment 2, time efficiency indicated a difference between sessions, such that participants solved trials faster in Session 2 than in Session 1. Furthermore, no session-by-gender effect was found using time efficiency. These two metrics were strongly correlated: in both experiments, $r \mathrm{~s} \geq .50, p \mathrm{~s}<.001$.
}

No differences were found between men and women in video game experience, $t(25.68)=.63, p=.53$, equal variances were not assumed, and including video game experience as a covariate did not appreciably affect these results.

\section{Landmark placement task ${ }^{4}$}

The landmark placement task for the second maze was scored out of 11 items, and participants correctly identified an average of $7.29(S D=2.87)$ landmarks. Accurately identifying the landmarks was highly correlated with path efficiency on this maze, $r(29)=-.46, p=.01$. Critically, accuracy on the landmark placement task was not significantly correlated with solution index for this maze, $r(29)=.29, p=.11$, nor with navigation success (i.e., number of trials on which the goal was reached), $r(29)=.34, p=.07$. Furthermore, men $(M=$ $8.44, S D=2.45)$ identified significantly more landmarks than did women $(M=5.73, S D=2.69), F(1,29)=8.06, p=.006$, $\eta_{\mathrm{p}}^{2}=.23$.

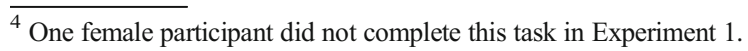




\section{Discussion}

In this experiment, participants first learned a virtual maze environment and were instructed to go to goal locations in retrieval trials, as in the normal DSP instructions, and then learned and navigated in a different virtual maze in which they were asked to take shortcuts on each trial. The results indicated that participants took more shortcuts in the second maze environment than in the first. Specifically, when asked to take shortcuts, 25 of the 32 participants showed an increase in solution index from Session 1 to Session 2, indicating that they were taking more shortcuts. This suggests that some participants who have the ability to take shortcuts prefer other navigation strategies when they are given more general instructions just to navigate to a goal. Interestingly, when given the shortcut instructions, the difference in solution indexes between men and women remained, and men were still more efficient than women. Furthermore, navigation strategy, as assessed by the number of successful shortcuts, was not correlated with ability to correctly place items on the landmark placement task. Overall, these results indicate that navigation strategies do not necessarily reflect ability to construct a survey (configural) representation of an environment.

\section{Experiment 2}

Although Experiment 1 indicated a large difference in shortcutting and efficiency when participants were asked to take shortcuts, instructions and order were confounded in that experiment, such that participants were always given the shortcut instructions second. The experiment also had relatively low power, since data were not collected for some trials. It is possible that, with practice on the task, participants learned to take more shortcuts, and thus the increase in shortcuts in the second session was due to practice rather than to the instructions. To address this potential confound, in Experiment 2 two groups of participants performed the DSP twice, in two different mazes but under the same instructions each time (either standard or shortcut instructions). With this manipulation, we are able to assess the effect of practice independently of the effect of instructions. Specifically, if the effects in Experiment 1 were due to practice, we would expect to find a main effect of session, such that everyone would take more shortcuts on the second maze, regardless of condition. However, if the type of instructions was driving the effect, then we would predict a main effect of condition, such that the participants asked to take shortcuts would take more shortcuts than would those in the control condition, for both experimental sessions. Again, as in Experiment 1 and the previous research, we expected a male advantage in navigation success (finding the goal) and efficiency, as well as more use of shortcuts by male participants.

\section{Method}

\section{Participants}

The participants were 87 University of California, Santa Barbara, undergraduates, who participated in return for course credit. Seven female participants were excluded from the analysis due to motion sickness $(n=6)$ or lack of English proficiency $(n=1)$. The analyses were based on data from 80 participants. Twenty males and 20 females participated in each condition. With this sample size and design, the power to detect a medium effect of instructions $\left(\eta_{\mathrm{p}}{ }^{2}=.07\right)$ at $p=.05$ was .80 .

\section{Design}

This experiment had a 2 (instruction type: original instructions vs. take shortcut) $\times 2$ (session: first vs. second set of trials $) \times 2$ (sex: male vs. female) experimental design. Session was manipulated within subjects, and instruction type, order, and sex were manipulated between subjects. The order of mazes (Maze 1 vs. Maze 2) was counterbalanced across participants.

\section{Materials}

The computer hardware and software were unchanged from Experiment 1. For each DSP test phase there were 20 trials, and data were collected on all trials.

\section{Procedure}

The procedure was the same as in Experiment 1, except that each participant was given the same instructions in both mazes (either standard "go to goal" or "take shortcut"). Trials were coded in the same manner as in Experiment 1.

\section{Results}

\section{Navigation performance}

Descriptive statistics can be found in Table 1. A 2 (session) $\times 2$ (gender) $\times 2$ (instruction condition) repeated measures ANOVA on success of navigation indicated that men $(M=$ $18.59, S D=1.50$ ) were significantly more successful than women $(M=16.69, S D=2.22), F(1,76)=28.88, p<.001$, $\eta_{\mathrm{p}}{ }^{2}=.28$. However, no other main effects or interactions were found, all $F \mathrm{~s}(1,76) \leq 2.33, p \mathrm{~s} \geq .13$.

\section{Navigation strategy}

A 2 ( (ession) $\times 2($ sex $) \times 2$ (instruction condition) repeated measures ANOVA on solution index indicated that the shortcut instruction group participants took significantly more shortcuts $(M$ 
$=.49, S D=.17)$ than the control group $(M=.32, S D=.19), F(1$, $76)=24.40, p<.001, \eta_{\mathrm{p}}{ }^{2}=.24$. Furthermore, as can be seen in Fig. 5, more shortcuts were taken in the second session $(M=.43$, $S D=.17)$ than in the first session $(M=.38, S D=.19), F(1,76)=$ $4.28, p=.04, \eta_{\mathrm{p}}{ }^{2}=.05$. This was a small effect, however, relative to the effect of instructions. As is shown in the dumbbell plots in Fig. 6, participants varied in both the size and direction of their strategy shifts across sessions. Although men $(M=.47, S D=.18)$ were more likely overall than women $(M=.34, S D=.17)$ to navigate by shortcuts, $F(1,76)=13.85, p<.001, \eta_{\mathrm{p}}{ }^{2}=.15$, none of the interactions were significant, $F_{\mathrm{S}}(1,76) \leq .3 .07$, all $p \mathrm{~s} \geq .08$.

\section{Navigation efficiency}

Analyses of path efficiency were conducted using a 2 (session) $\times 2(\mathrm{sex}) \times 2$ (instruction condition) repeated measures ANOVA (see note 3 ). Here, the participants in the shortcut condition $(M=2.27, S D=.37)$ navigated more efficiently than those in the control condition $(M=2.52, S D=.47), F(1,76)=$ $10.89, p=.001, \eta_{\mathrm{p}}{ }^{2}=.13$. However, no differences in the measure of efficiency were found between sessions, $F(1,76)$ $=1.98, p=.16$. A main effect of sex was found, $F(1,76)=7.58$, $p=.007, \eta_{\mathrm{p}}{ }^{2}=.09$, such that men $(M=2.29, S D=.43)$ navigated more efficiently than women $(M=2.50, S D=.41)$. The Session $\times$ Gender interaction was significant, $F(1,76)=$ $4.24, p=.04, \eta_{\mathrm{p}}{ }^{2}=.05$, indicating that men gained more in path efficiency in the second session, $F(1,76)=6.10, p=.02, \eta_{\mathrm{p}}{ }^{2}=$ .07 , than did women, $F(1,76)=.21, p=.65$. No other interactions were significant, all $F_{\mathrm{s}}(1,76) \leq .39, p \mathrm{~s} \geq .53$. A sex difference was found in video game experience, $t(71.42)=$ 5.07, $p<.001$, and equal variances were not assumed, such that men reported playing more video games $(M=3.15, S D=$ 1.46) than did women $(M=1.70, S D=1.07)$. Entering frequency of video game play as a covariate in this ANOVA eliminated the Session $\times$ Gender interaction, but it preserved the main effects of both gender, $F(1,75)=4.00, p=.05, \eta_{\mathrm{p}}{ }^{2}=$ .05 , and condition, $F(1,75)=10.50, p=.002, \eta_{\mathrm{p}}{ }^{2}=.12$.

\section{Landmark placement task}

Participants correctly placed $6.84(S D=3.04)$ of the 11 landmarks. A univariate ANOVA on maze reconstruction accuracy indicated no significant difference between conditions, $F(1,76)$ $=.12, p=.73$, and no interaction between condition and gender,

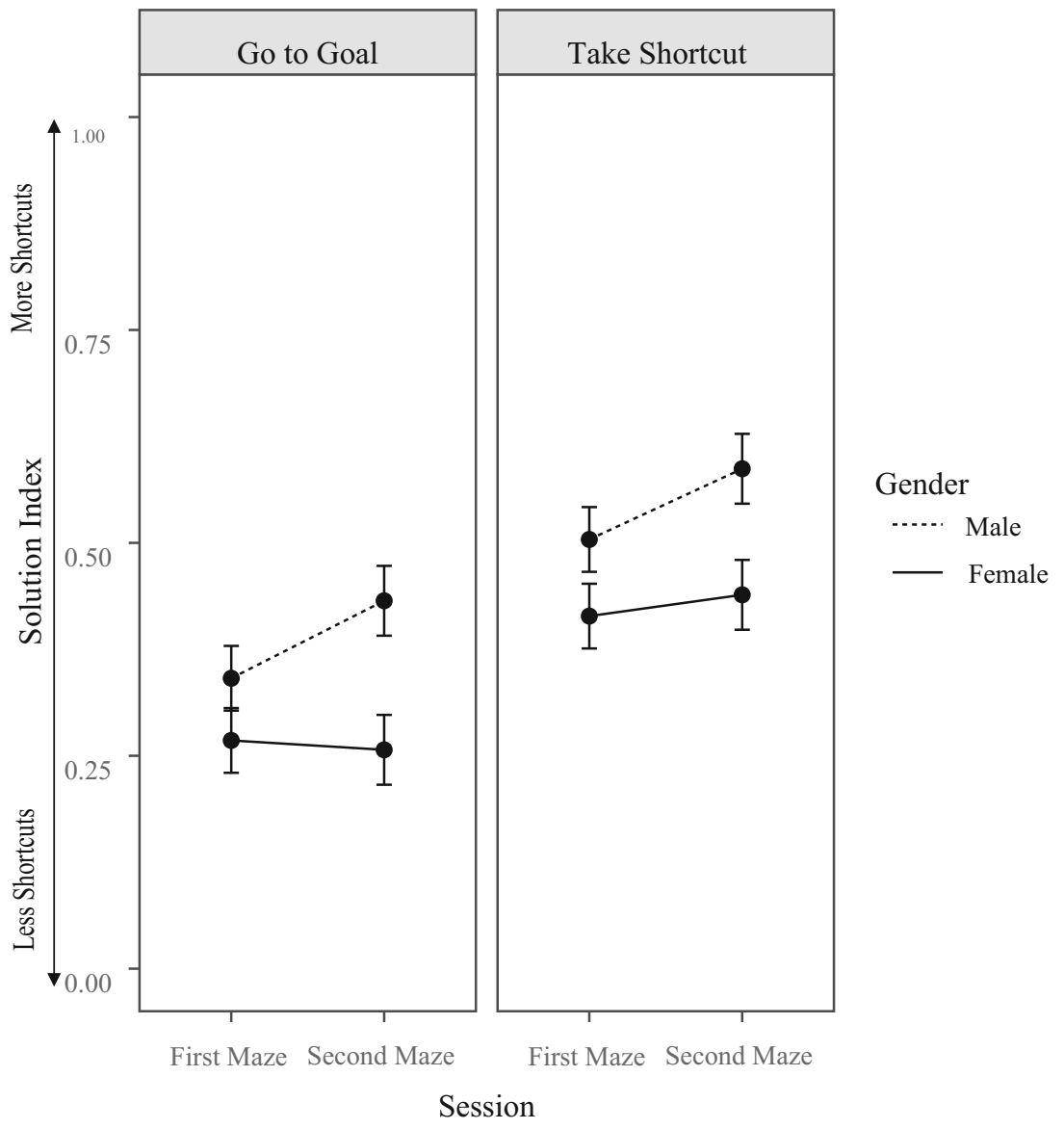

Fig. 5 Solution index by type of instructions, session, and gender in Experiment 2. Error bars indicate standard errors. Larger values of the solution index indicate more shortcuts 


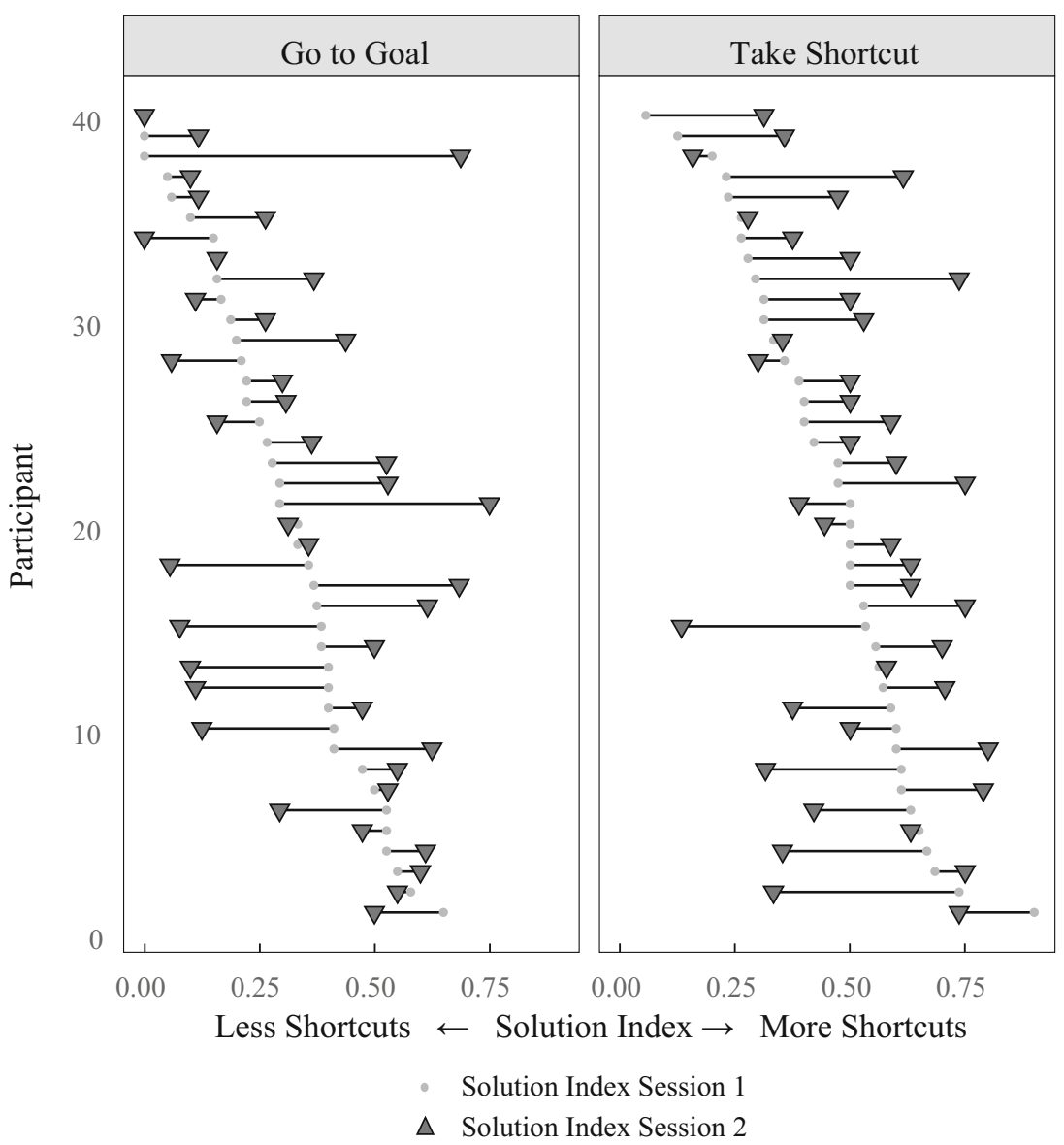

Fig. 6 Dumbbell plot showing changes in solution index as a function each session in each condition in Experiment 2. The gray points represent each participant's solution index in the first session, and the gray triangles represent the solution index in the second session

$F(1,76)=.54, p=.46$. As in Experiment 1 , men $(M=7.93, S D=$ 2.84) identified more landmarks than did women $(M=5.75, S D$ $=2.87), F(1,76)=11.40, p=.001, \eta_{\mathrm{p}}^{2}=.13$.

Correlations between objective measures in the DSP (solution index, success, and path efficiency) and the landmark placement task were computed for each condition separately. As in Experiment 1, maze accuracy was not significantly correlated with solution index in either the control condition, $r(38)$ $=-.05, p=.77$, or the shortcut condition, $r(38)=.25, p=.12$. In both conditions, maze reconstruction accuracy was significantly correlated with success in reaching the goal [both $r \mathrm{~s}(38) \geq$ .59 , both $p \mathrm{~s}<.001]$. However, contrary to Experiment 1 , maze reconstructions did not correlate significantly with path efficiency in either condition, both $r \mathrm{~s}(38) \leq-.21$, both $p \mathrm{~s} \geq .19$.

\section{Discussion}

In this experiment, two groups of participants navigated in virtual maze environments under the same instructions twice. This design allowed us to separate the effects of practice from the effects of task instructions. Although there was a significant effect of practice, such that participants took more shortcuts on the second set of trials, it is notable that the effect of instructions on solution indexes was substantially larger. Therefore, the instructions played an important role in whether people took shortcuts. Furthermore, as in previous research and Experiment 1, men took more shortcuts when given either the standard instructions or the shortcut instructions for this task. Men navigated more efficiently than women, and adding video game experience as a covariate did not reduce this sex difference, suggesting that it was not merely due to more experience with virtual environments. As in Experiment 1, navigation strategy was not correlated with ability to correctly place landmarks on the landmark placement task. Moreover, landmark placement did not depend on the task instructions.

Overall, the results of this experiment show that people have more competence to take shortcuts than they demonstrate when they are given standard instructions to navigate to a goal location.

\section{General discussion}

Across two experiments, using different experimental designs, we examined whether different strategies in the dual-solution paradigm (Marchette et al., 2011) reflect differences in the ability to construct and subsequently utilize knowledge of 
the configuration of the environment, or differences in choosing whether to use such knowledge. In Experiment 1, participants navigated with standard instructions ("please navigate to the harp") and then navigated in a second learned environment with shortcut instructions ("please take the shortest path to the swing"). Participants took more shortcuts and more efficient paths during the second session. In Experiment 2, a between-subjects design was utilized in which two groups of participants were given the same instructions in successive sessions. Here, the instructions had a large effect, and practice had a relatively small effect on strategy choices.

\section{Ability versus strategy}

This investigation focused on the distinction between competence, or what you can do, and performance, or what you actually do, in a navigation task. In the literature on navigation, taking shortcuts is considered to depend on both having and using survey (configural) knowledge of the environment (Down \& Stea, 1973; Golledge, 1999), which must be inferred from route experience. If the ability to construct survey knowledge from experience of a route is the only determinant of taking a shortcut rather than a route strategy in the DSP, then navigation strategy should not be influenced by task instructions, but should instead be determined by the quality of a person's survey knowledge. The fact that people took more shortcuts when instructed to do so suggests that differences in performance (in this case, taking a shortcut) do not always reflect differences in competence (i.e., the ability to take a shortcut). Furthermore, the landmark placement task, a measure of the completeness of environmental knowledge, does not correlate with strategy as assessed by the DSP, and performance on landmark placement was not affected by navigation instructions. Overall, the results presented here suggest that using a shortcut strategy in the DSP is not only influenced by the quality of one's representation of the environment, but also by the specific task instructions.

Although the landmark placement task is a measure of the quality (completeness) of a person's knowledge of an environment, we acknowledge that it is not necessarily a measure of survey knowledge. This task can be effectively completed with either survey or route knowledge, in that one could complete the task with route knowledge by placing the landmarks in the order of the route. An important direction for future research will be to measure survey knowledge of the environment after learning, possibly with a pointing task, to more directly assess the relation between survey knowledge and navigation strategy. For example, cognitive graph theory (Chrastil \& Warren, 2015) proposes that graph knowledge (of local connections between landmarks in a known environment) may be sufficient to find shortcuts, so that metrically accurate survey knowledge is not necessary for using a shortcut strategy.

It is perhaps not surprising that even with accurate environmental knowledge, someone might not take a shortcut on a given trial. There are a number of reasons why a person might decide to take a learned route instead of a shortcut, which is more efficient. First, during the learning phase, participants always took the same route. This could have led some participants to believe that their task was to take that route in the test phase. Anecdotally, throughout the test phase with control instructions, participants sometimes asked whether they could go anywhere in the maze (to which we answered "Do whatever you want to do"), indicating that they were unsure whether they were allowed to take a route other than the learned route. Second, given the 40-s time limit on each trial, participants might have been hesitant or reluctant to take a potentially risky shortcut if they were unsure that it would bring them to their goal within the time limit. The unwillingness to take shortcuts in uncertain situations may be linked to confidence in the ability to do so. Third, participants might have taken the learned route because it was less cognitively demanding. The present research cannot distinguish between these possibilities, and this will be an important direction for future research.

It is also possible that the large shift in shortcut use was driven by a class of navigators intermediate between those who could infer good survey knowledge from route experience and those who could only construct route representations. This intermediate group might be classed as "reluctant shortcutters," who do not have enough confidence in their survey knowledge to take shortcuts spontaneously, but could take a shortcut when they were asked to do so. This group of people might be akin to the "nonintegrator" group of navigators found by Weisberg and Newcombe (2016), who are able to point to the locations on a route but not between routes, suggesting that such navigators have local but not global survey knowledge. The small increase in taking shortcuts due to practice alone (found in Exp. 2) also suggests that people might develop more confidence or more configural knowledge of an environment on the fly, during the test trials.

More generally, when considering navigating the real world, it makes intuitive sense that your preferred strategy would not necessarily be taking the shortest or most efficient route. There are instances in which taking a shortcut between locations is neither the best strategy nor advisable. For instance, when navigating the city at night, a dark alley might provide the shortest path to the location of interest, but of course should not be taken. Flexible use of different navigation strategies, in general, may be more adaptive.

\section{Sex differences}

Recent work using the DSP has shown that men were more likely than women to take shortcuts (Boone et al., 2018), a result that is consistent with self-report studies suggesting that women prefer route strategies (Lawton, 1994). Although women took more shortcuts when instructed to do so, the sex difference remained in the shortcut conditions of both experiments. 
Overall, these results indicate that, although women are able to take shortcuts, they may need additional incentives to use this strategy. For example, women may be less likely to take a risk on taking a shortcut, or may prefer the learned route because it is less cognitively demanding. Recent evolutionary psychology studies have shown that during exploration of an environment, women are more likely to revisit a location and pause, effects that have been attributed to risk avoidance (Gagnon, Cashdan, Stefanucci, \& Creem-Regehr, 2016). In a real-world scenario, the risks involved in taking unknown paths could be life-threatening, especially for women, and this might lead to a female preference for taking well-known paths.

It should also be noted that typical sex differences in efficiency and success in reaching the goal (e.g., Collucia \& Louse, 2004; Moffat et al., 1998; Sandstrom et al., 1998) were observed, in favor of males. Given that each trial had a time limit, it is likely that differences in reaching the goal also reflected differences in efficiency. A commonly proposed explanation of such effects is that males are more efficient in navigating virtual environments because they have more video game experience (Lucas \& Sherry, 2004; Ogletree \& Drake, 2007). In our study, entering frequency of video game play as a covariate did not affect the results appreciably. However, it should be acknowledged that our measure of video game play was just a one-item measure that asked about frequency of play. Future research should use a more extensive and sensitive measure of participants' experience with spatial video games in virtual environments, which would give them the type of experience that might transfer to navigation tasks.

Finally, in both experiments, men outperformed women in the landmark placement task. Although this task can be completed effectively with either route or survey knowledge, it suggests that women had less complete knowledge of the environment following their learning experience. Weisberg and Newcombe (2016) also found a sex difference in a layout reconstruction task, whereas other research has found no sex differences in a layout reconstruction task (Weisberg et al., 2014) or in sketch mapping (Coluccia \& Louse, 2004). Clearly, more research is needed to understand the situations under which sex differences in spatial learning do and do not occur.

It is important to acknowledge that the present research was conducted in a desktop virtual environment, in which there is no additional physical effort when one takes a longer path, and in which some visual and self-motion cues used in navigation were not present (Hegarty, Montello, Richardson, Ishikawa, \& Lovelace, 2006; Waller, Loomis, \& Haun, 2004; Waller, Loomis, \& Steck, 2003). Future work will need to assess the extent to which navigation strategy and ability in the DSP relates to navigation in the real world.

\section{Conclusion}

We examined the sources of individual differences in navigation strategy bias. This research indicated that differences in navigation strategy should not be interpreted as differences in the ability to construct and use configural or survey-based spatial knowledge, but are instead affected by instructions. Differences in navigation strategy as measured by the DSP reflect factors other than differences in the ability to take shortcuts.

Author note The authors thank Xinyi Gong and Lindsey Nguyen for assistance in the development of code, and Shaina Michalowicz for assistance with data collection. We also thank Elizabeth R. Chrastil, Chuanxiuyue $\mathrm{He}$, and three anonymous reviewers for providing critical comments on an earlier version of the manuscript. This work was supported by a Challenge grant through the California Nanosystems Institute at the University of California, Santa Barbara. The data for both experiments are available at https://osf.io/ykxts/. Neither experiment was preregistered.

\section{References}

Boone, A. P., Gong, X., \& Hegarty, M. (2018). Sex differences in navigation strategy and efficiency. Memory \& Cognition, 46, 909-922. https://doi.org/10.3758/s13421-018-0811-y

Chomsky, N., \& Halle, M. (1965). Some controversial questions in phonological theory. Journal of Linguistics, 1, 97-138.

Chrastil, E. R., \& Warren, W. H. (2015). Active and passive spatial learning in human navigation: Acquisition of graph knowledge. Journal of Experimental Psychology: Learning, Memory, and Cognition, 41, $1162-1178$.

Coluccia, E., \& Louse, G. (2004). Gender differences in spatial orientation: A review. Journal of Environmental Psychology, 24, 329-340.

Downs, R. M., \& Stea, D. (1973). Cognitive maps and spatial behavior: Process and products. In R. M. Downs \& D. Stea (Eds.), Image and environment: Cognitive mapping and spatial behavior (pp. 8-26). Chicago: Aldine.

Furman, A. J., Clements-Stephens, A. M., Marchette, S. A., \& Shelton, A. L. (2014). Persistent and stable biases in spatial learning mechanisms predict navigational style. Cognitive, Affective, \& Behavioral Neuroscience, 14, 1375-1391. https://doi.org/10.3758/s13415-0140279-6

Gagnon, K. T., Cashdan, E. A., Stefanucci, J. K., \& Creem-Regehr, S. H. (2016). Sex differences in exploration behavior and the relationship to harm avoidance. Human Nature, 27, 82-97.

Golledge, R. G. (1999). Wayfinding behavior: Cognitive mapping and other spatial processes. Baltimore: Johns Hopkins University Press.

Hartley, T., Maguire, E. A., Spiers, H. J., \& Burgess, N. (2003). The wellworn route and the path less traveled: Distinct neural bases of route following and wayfinding in humans. Neuron, 37, 877-888.

Hegarty, M., Montello, D. R., Richardson, A. E., Ishikawa, T., \& Lovelace, K. (2006). Spatial abilities at different scales: Individual differences in aptitude-test performance and spatial-layout learning. Intelligence, 34, 151-176. 
Iaria, G., Petrides, M., Dagher, A., Pike, B., \& Bohbot, V. D. (2003). Cognitive strategies dependent on the hippocampus and caudate nucleus in human navigation: variability and change with practice. Journal of Neuroscience, 23, 5945-5952.

Ishikawa, T., \& Montello, D. R. (2006). Spatial knowledge acquisition from direct experience in the environment: Individual differences in the development of metric knowledge and the integration of separately learned places. Cognitive Psychology, 52, 93-129.

Lawton, C. A. (1994). Gender differences in way-finding strategies: Relationship to spatial ability and spatial anxiety. Sex Roles, 30, 765-779.

Lucas, K., \& Sherry, J. L. (2004). Sex differences in video game play: A communication-based explanation. Communication Research, 31, 499-523.

Maguire, E. A., Burgess, N., Donnett, J. G., Frackowiak, R. S., Frith, C. D., \& O'Keefe, J. (1998). Knowing where and getting there: A human navigation network. Science, 280, 921-924.

Maguire, E. A., Woollett, K., \& Spiers, H. J. (2006). London taxi drivers and bus drivers: A structural MRI and neuropsychological analysis. Hippocampus, 16, 1091-1101. https://doi.org/10.1002/hipo.20233

Marchette, S. A., Bakker, A., \& Shelton, A. L. (2011). Cognitive mappers to creatures of habit: Differential engagement of place and response learning mechanisms predicts human navigational behavior. Journal of Neuroscience, 31, 15264-15268.

Moffat, S. D., Hampson, E., \& Hatzipantelis, M. (1998). Navigation in a "virtual" maze: Sex differences and correlation with psychometric measures of spatial ability in humans. Evolution and Human Behavior, 19, 73-87.

O'Keefe, J., \& Nadel, L. (1978). The hippocampus as a cognitive map. Oxford: Oxford University Press, Clarendon Press.
Ogletree, S. M., \& Drake, R. (2007). College students' video game participation and perceptions: Gender differences and implications. Sex Roles, 56, 537-542.

Sandstrom, N. J., Kaufman, J., \& Huettel, S. A. (1998). Differential cue use by males and females in a virtual environment navigation task. Cognitive Brain Research, 6, 351-360.

Schinazi, V. R., Nardi, D., Newcombe, N. S., Shipley, T. F., \& Epstein, R. A. (2013). Hippocampal size predicts rapid learning of a cognitive map in humans. Hippocampus, 23, 515-528.

Waller, D., Loomis, J. M., \& Haun, D. B. M. (2004). Body-based senses enhance knowledge of directions in large-scale environments. Psychonomic Bulletin \& Review, 11, 157-163. https://doi.org/10. 3758/BF03206476

Waller, D., Loomis, J. M., \& Steck, S. D. (2003). Inertial cues do not enhance knowledge of environmental layout. Psychonomic Bulletin \& Review, 10, 987-993.

Weisberg, S. M., \& Newcombe, N. S. (2016). How do (some) people make a cognitive map? Routes, places, and working memory. Journal of Experimental Psychology: Learning, Memory, and Cognition, 42, 768-785.

Weisberg, S. M., Schinazi, V. R., Newcombe, N. S., Shipley, T. F., \& Epstein, R. A. (2014). Variations in cognitive maps: Understanding individual differences in navigation. Journal of Experimental Psychology: Learning, Memory, and Cognition, 40, 669-682. https://doi.org/10.1037/a0035261

Publisher's note Springer Nature remains neutral with regard to jurisdictional claims in published maps and institutional affiliations. 